\title{
Engaging older adults as partners in transitional care research
}

\author{
Rebecca Ganann RN PhD, Carrie McAiney PhD, William Johnson
}

Cite as: CMAJ 2018;190(Suppl 1):S40-S41. doi: 10.1503/cmaj.180396

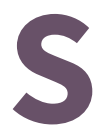

ystematic reviews have found that older adults with multimorbidity (multiple chronic conditions) are at increased risk for depression and poorer quality of life. ${ }^{1}$ Furthermore, the presence of multimorbidity results in substantial health care spending. ${ }^{2}$ Older adults with multimorbidity who are transitioning home after a hospital admission are particularly vulnerable to fragmented care, as they typically receive services from numerous providers who lack a common system for coordination and communication. There is limited evidence on how best to provide well-coordinated transitional care to older adults with multimorbidity and depressive symptoms. ${ }^{3-5}$

The Community Assets Supporting Transitions (CAST) study, a pragmatic effectiveness-implementation randomized controlled trial (ClinicalTrials.gov NCT03157999), is designed to test a support program for older adults living with multimorbidity and depressive symptoms who are being discharged from hospital to home. It builds on pilot feasibility and effectiveness work, ${ }^{6}$ and aims to improve care transitions in older adults with multimorbidity and depressive symptoms through adapting and testing a six-month support program. Estimated enrolment across study sites is 216 participants. The trial's intervention is delivered by a registered nurse through home visits and phone calls tailored to individual patient needs. Core components include care coordination and system navigation, medication review, assessment of needs and risks, problem-solving therapy, and patient and caregiver education, in addition to usual care. Control participants receive usual care. The study explores program effects on health outcomes and costs for older adults and their caregivers; how the program is adapted and implemented in three Ontario communities (Hamilton, Burlington and Sudbury); and what will be required to sustain and scale up the program in other communities.

This integrated knowledge translation study, in which multiple stakeholders are involved in designing, implementing and evaluating the intervention, is based on the Knowledge-to-Action Framework. ${ }^{7}$ Our approach to patient engagement is aligned with this framework, relying on meaningful engagement of key stakeholders and valuing the integral role that patients play. Patient and caregiver partners have been integrated throughout the study's governance structure and strategically engaged using multiple mechanisms, ${ }^{8}$ including serving as co-investigators and members of the local community advisory boards.

\section{KEY POINTS}

- The Community Assets Supporting Transitions study, a pragmatic effectiveness-implementation randomized controlled trial, is designed to test a support program for older adults living with multimorbidity and depressive symptoms who are being discharged from hospital to home.

- Incorporating the experiences and skills of patient and caregiver partners in hospital-to-home transitional care research can inform and enhance all stages of the research process and enrich researchers' understanding of the topic.

- In working with patient partners with multimorbidity, and those caring for them, researchers have needed to be mindful of the potential burden of research-related requests, and have learned the importance of maintaining contact during study quiet times.

The patient-engagement approach in this study was guided by the Canadian Institutes of Health Research Patient Engagement Framework, ${ }^{8}$ strategically engaging patients and caregivers to ensure research alignment with patients' values and priorities; reflect their experiences with the health system; situate the intervention within local resources and address gaps; and identify patient- and caregiver-relevant health and system experience outcomes. Older adults and caregivers have been recruited from all study sites, with the assistance of local stakeholders, as patient and caregiver partners. Patient and caregiver partners have informed adaptations to the study intervention, identified patient- and caregiver-relevant outcome and experience measures, informed recruitment and consent processes, and contributed to the study's knowledge translation plan and products (e.g., presentations, videos and journal manuscript).

Patient and caregiver partners have also helped researchers and trainees to better understand issues that older adults face with transitional care. Patient and caregiver partners have expressed value in the opportunity to "share experiences in a meaningful way to help others" and have expressed the importance of "knowing that someone is addressing these problems" (i.e., managing multimorbidity and gaps in communication and service coordination).

An important contribution of the CAST study is the engagement of vulnerable older adults as patient and caregiver partners, 
an understudied subgroup in the patient-engagement literature. ${ }^{9}$ Patients' complex, and sometimes fragile, health status can make engagement challenging, as can the demands placed on their caregivers. We have gained many valuable insights regarding the engagement of older adult patients and their caregivers as research partners. One patient partner expressed, "when starting to partner, it is important to clarify roles; that the [patient] is a partner in the research team, which is different than if patients or caregivers have been research participants in the past." Several partners have identified the importance of researchers investing time to understand their stories, ensure clarity of purpose and expectations (i.e., what is being asked of partners), and ensure partners do not feel rushed or inhibited in any way. Having an identified contact within the team and building personal connections have also been important.

Patient partners who are members of the research team have identified the benefit of having researchers help them to understand how their role and experiences can inform the research. In working with patient partners with multimorbidity, or with those who are caring for them, researchers have needed to be mindful of the potential burden of research-related requests. At the same time, it is important not to make assumptions about what contributions patient and caregiver partners can make. Thus, it is important to communicate regularly with patient and caregiver partners and tailor their engagement based on their individual needs and preferences. For example, determining preferences for communication approaches (in person v. telephone meetings, email v. postal mail) and being flexible to accommodate changes in health status or caregiving demands is important.

This pragmatic trial, coordinated from and supported by the Aging, Community and Health Research Unit, is currently being implemented in three study communities. Time delays associated with slower-than-anticipated recruitment and delayed ethics approval have challenged us. Strong early engagement and enthusiasm were achieved with our patient and caregiver partners; however, delays have created challenges in maintaining engagement during study "quiet" times. Patient and caregiver partners have provided valuable feedback about continuity of communication to keep the study "fresh" in their minds, including the value of sharing updates about challenges, as it is sometimes hard to get back up to speed about the study when there have been substantial delays. Ensuring ongoing, mutual understanding is key to effective collaborations.

A formal evaluation of the implementation and impact of the study's patient-engagement strategy is in its early stages; however, the value of partnering with patients and caregivers in research has been seen in terms of strengthening study approaches and positive impacts on researchers. The CAST study engages in a variety of activities to understand diverse patient and caregiver partner perspectives. Patient and caregiver partners have expressed feeling that their contributions are heard and valued by the research team. As our patient-partner coauthor stated, "We're all [as older adults] running into similar situations but may differ based on particular health situations ... that's the beauty of the research ... bringing those perspectives together."

\section{References}

1. Marengoni A, Angleman S, Melis R, et al. Aging with multimorbidity: a systematic review of the literature. Ageing Res Rev 2011;10:430-9.

2. Sambamoorthi U, Tan X, Deb A. Multiple chronic conditions and healthcare costs among adults. Expert Rev Pharmacoecon Outcomes Res 2015;15:823-32.

3. Bryant-Lukosius D, Carter N, Reid K, et al. The clinical effectiveness and costeffectiveness of clinical nurse specialist-led hospital to home transitional care: a systematic review. J Eval Clin Pract 2015;21:763-81.

4. Rennke S, Nguyen OK, Shoeb MH, et al. Hospital-initiated transitional care interventions as a patient safety strategy: a systematic review. Ann Intern Med 2013;158:433-40.

5. Kansagara D, Chiovaro JC, Kagen D, et al. Transitions of care from hospital to home: an overview of systematic reviews and recommendations for improving transitional care in the veterans health administration. VA Evidence-based Synthesis Program (ESP) reports. Washington (DC): Department of Veterans Affairs; 2015.

6. Markle-Reid M, McAiney C, Forbes D, et al. An interprofessional nurse-led mental health promotion intervention for older home care clients with depressive symptoms. BMC Geriatr 2014;14:62.

7. Graham ID, Logan J, Harrison MB, et al. Lost in knowledge translation: Time for a map? J Contin Educ Health Prof 2006;26:13-24.

8. Strategy for Patient Oriented Research - Patient Engagement Framework. Ottawa: Canadian Institute of Health Research; (modified 2014 July 2). Available: www.cihr-irsc.gc.ca/e/48413.html\#a7 (accessed 2018 Jan. 5).

9. Naylor MD, Hirschman KB, O'Connor M, et al. Engaging older adults in their transitional care: What more needs to be done? J Comp Eff Res 2013;2:457-68.
More information on this project is available at www.ossu.ca/ IMPACTAwards.

\section{Competing interests: None declared.}

This article was solicited and has been peer reviewed.

Affiliations: School of Nursing, McMaster University (Ganann), Hamilton, Ont.; School of Public Health and Health Systems, University of Waterloo (McAiney), Waterloo, Ont.; patient partner (Johnson), Coburg, Ont.

Contributors: All authors contributed to the conception and design of the work, drafted the manuscript, revised it critically for important intellectual content, gave final approval of the version to be published and agreed to be accountable for all aspects of the work.

Funding: This project is supported by grants from OSSU (the Ontario SPOR [Strategy for Patient-Oriented Research] SUPPORT [Support for People and Patient-Oriented Research and Trials] Unit) and the Labarge Optimal Aging Initiative.
Acknowledgements: The authors thank the Community Assets Supporting Transitions research team members, specifically Dr. Maureen Markle-Reid (co-principal investigator), Ruta Valaitis and Jenny Ploeg, the Aging, Community and Health Research Unit research administrator, Laurie Kennedy, and coordinators Agata Drozd and Tracey Chambers. The authors also sincerely thank the research team patient partners (Gail Heald-Taylor, Larry Ogden and Pat Reid) and the local Community Advisory Board members (patient and public partners, and health and social service providers) for their ongoing support for the study and valuable contributions to research design and implementation. The authors continue to grow in their understanding of how to meaningfully partner with patients and iteratively refine their strategies with constructive input from their patient and public partners.

Correspondence to: Rebecca Ganann, ganann@mcmaster.ca 